\title{
Cardinal Interpolation by Multivariate Splines
}

\author{
By C. K. Chui,* K. Jetter, and J. D. Ward
}

\begin{abstract}
The purpose of this paper is to investigate cardinal interpolation using locally supported piecewise polynomials. In particular, the notion of a commutator is introduced and its connection with the Marsden identity is observed. The order of a commutator is shown to be equivalent to the Strang and Fix conditions that arise in the study of the local approximation orders using quasi-interpolants. We also prove that scaled cardinal interpolants give these local approximation orders.
\end{abstract}

Introduction. Cardinal interpolation by bivariate box splines was first studied by de Boor, Höllig, and Riemenschneider [3]. The purpose of our paper is to investigate the cardinal interpolation problem from a different point of view. In particular, the notion of a commutator is introduced. It will be shown that this notion generalizes the Marsden identity for univariate splines to the multivariate setting. The order of a commutator will be shown to be equivalent to the Strang and Fix conditions used in the study of the order of controlled approximation by Dahmen and Micchelli [8] or local approximation by de Boor and Jia [4]. An application to obtain approximation orders through the constructive method of scaled cardinal interpolation will also be studied in this paper.

1. Preliminaries. This section consists of preliminary material for multivariate cardinal interpolation. Our approach is motivated by the work in [5] and [13] where cardinal interpolation in $l_{2}$ was connected with certain convolution operators $L_{\phi}$.

Let $Z$ denote the set of integers and $Z_{+}$, the nonnegative ones. For any given complex sequence $\phi \equiv\left(\phi_{j}\right)_{j \in Z^{N}} \in l_{1}\left(Z^{N}\right)$ we denote the discrete Fourier transform of $\phi$ by

$$
\tilde{\phi}(\xi):=\sum_{j \in Z^{N}} \phi_{j} e^{i j \cdot \xi}, \quad \xi \in R^{N}
$$

and extend $\tilde{\phi}(\xi)$, at least formally, to all of $\mathbf{C}^{N}$ by defining

$$
\sigma_{\phi}(z)=\sum_{j \in Z^{N}} \phi_{j} z^{j}
$$

Received September 19, 1985; revised March 11, 1986.

1980 Mathematics Subject Classification. Primary 41A05, 41A15; Secondary 41A63.

Key words and phrases. Cardinal interpolation, scaled cardinal interpolation, Fourier transform, discrete Fourier transform, box splines, Marsden identity.

* The research of this author was supported by the U.S. Army Research Office under Grant No. DAAG 29-84-K-0154. 
where the usual multivariate notation $z^{j}=z_{1}^{j_{1}} \cdots z_{N}^{j_{N}}, j=\left(j_{1}, \ldots, j_{N}\right), \quad z=$ $\left(z_{1}, \ldots, z_{N}\right)$, is used. Since the series that defines $\tilde{\phi}$ is absolutely and uniformly convergent, $\tilde{\phi}$ is a continuous function and $2 \pi$-periodic with respect to each variable. Moreover, any $\phi=\left\{\phi_{j}\right\}_{j \in Z^{N}} \in l_{1}\left(Z^{N}\right)$ also defines a convolution operator on $l_{p}, 1 \leqslant p \leqslant \infty$, given by $v=L_{\phi}(\mu)$ where $\mu=\left\{\mu_{j}\right\}, v=\left\{v_{j}\right\}$, and

$$
v_{j}=\sum_{k \in Z^{N}} \phi_{j-k} \mu_{k}, \quad j \in Z^{N} \text {. }
$$

More generally, for a given continuous $\phi$ on $R^{N}$ having compact support,

$$
L_{\phi}(\mu)(x):=\sum_{k \in Z^{N}} \phi(x-k) \mu_{k}
$$

defines a continuous function on $R^{N}$ whose restriction to $Z^{N}$ gives rise to a map from $l_{p}\left(Z^{N}\right)$ into itself. Hereafter, $L_{\phi}(\mu)$ will denote either a function on $R^{N}$ or a map on $l_{p}$ depending on context. In the case $L_{\phi}(\cdot)$ is a map on $l_{p}\left(Z^{N}\right), L_{\phi}(\cdot)$ is a bounded linear transformation and

$$
\left\|L_{\phi}(\mu)\right\|_{p} \leqslant\|\phi\|_{1}\|\mu\|_{p}, \quad 1 \leqslant p \leqslant \infty .
$$

In the following we shall deal with sequences with finite support, $\left.\phi\right|_{Z^{N}}$, where $\phi$ : $R^{N} \rightarrow R$ is a piecewise polynomial function with compact support. For short, we call such $\phi$ a locally supported spline, or ls-spline. Under these assumptions, $\tilde{\phi}$, as defined above, is a trigonometric polynomial.

Consider the following problem of cardinal interpolation with translates of $\phi$ : Given the data $v \in l_{p}\left(Z^{N}\right)$, determine the existence and uniqueness of a sequence $\mu \in l_{p}\left(Z^{N}\right)$ such that $L_{\phi}(\mu)(x)$ interpolates the data $v$, i.e., $\left.L_{\phi}(\mu)\right|_{Z^{N}}=v$. If the answer is affirmative, we will say that the problem is $l_{p}$-solvable (for $p=\infty$, de Boor et al. [3] use the notion "correct"). It turns out that the problem of cardinal interpolation with translates of $\phi$ is $l_{p}$-solvable if and only if the corresponding convolution operator $L_{\phi}: l_{p} \rightarrow l_{p}$ is invertible. Indeed, the requirement that any $l_{p}$ sequence can be interpolated uniquely requires $L_{\phi}$ to be one-to-one and onto. Since $L_{\phi}$ is continuous, an application of the Banach inverse theorem shows that $L_{\phi}^{-1}$ is bounded. The following lemma, which is related to [5, Theorem 7] will be needed in Sections 2 and 3.

LEMMA 1.1. For any $\phi \in l_{1}\left(Z^{N}\right)$ the following statements are equivalent:

(i) $L_{\phi}: l_{2} \rightarrow l_{2}$ has a continuous inverse.

(ii) $L_{\phi}: l_{p} \rightarrow l_{p}$ has a continuous inverse for all $p, 1 \leqslant p \leqslant \infty$.

(iii) The symbol $\sigma_{\phi}$ of $\phi$ does not vanish on $T^{N}:=\left\{\left(z_{1}, \ldots, z_{N}\right) \in \mathbf{C}^{N}:\left|z_{i}\right|=1\right.$, $i=1, \ldots, N\}$.

The proof of the above lemma involves standard arguments using Fourier transform techniques.

Remark. It is not hard to verify that $L_{\phi}: l_{2} \rightarrow l_{2}$ is a symmetric operator if and only if $\phi$ is Hermitian (i.e., $\phi_{-j}=\bar{\phi}_{j}, j \in Z^{N}$ ). In this case, $\tilde{\phi}$ is a real function, continuous and $2 \pi$-periodic with respect to each variable, and condition (iii) of Lemma 1.1 may be written as

$$
\operatorname{sgn} \tilde{\phi}(0) \cdot \tilde{\phi}(\xi) \geqslant C_{\phi}>0 \text { for all } \xi \in[-\pi, \pi]^{N} .
$$


Using Lemma 1.1 and the characterization of $l_{\infty}$-solvability which appeared in [3] and [7], the following can be established.

Proposition 1.1. Let $\phi: R^{N} \rightarrow R$ be a piecewise polynomial function with compact support. Then the following statements are equivalent:

(i) The problem of cardinal interpolation with translates of $\phi$ is $l_{2}$-solvable.

(ii) The problem of cardinal interpolation with translates of $\phi$ is $l_{p}$-solvable for all $p$, $1 \leqslant p \leqslant \infty$.

(iii) The symbol $\sigma_{\phi}$ corresponding to $L_{\phi}$ does not vanish on $[-\pi, \pi]^{N}$.

(iv) The problem of cardinal interpolation with translates of $\phi$ is $l_{\infty}$-solvable.

2. The Fundamental Functions. This section is devoted to extending Schoenberg's univariate results [11] on characterizing the cardinal spline interpolant of $L=\left(\delta_{0, i}\right)$, where $\delta_{i, j}$ denotes the Kronecker symbol. In particular, the exponential decay of the "spline" solution is obtained for the multivariate case.

Hereafter, assume that the symbol $\sigma_{\phi}$ does not vanish on $T^{N}$, so that $|\tilde{\phi}(\xi)| \geqslant C_{\phi}$ $>0$ on $R^{N}$. This follows since $\tilde{\phi}(\xi)=\sum_{j} \phi_{j} e^{i j \cdot \xi}=\left.\sigma_{\phi}(z)\right|_{T^{N}}$. By Lemma 1.1, there is one and only one sequence $\lambda \in l_{1}\left(Z^{N}\right)$ satisfying

$$
\delta_{0, i}=\sum_{j} \phi_{i-j} \lambda_{j}, \quad i \in Z^{N} .
$$

In addition, for the sequence $\delta:=\left(\delta_{0, i}\right)_{i \in Z^{N}}$, we have $1=\tilde{\delta}=\tilde{\phi} \tilde{\lambda}$ and, hence,

$$
\lambda_{j}=\frac{1}{(2 \pi)^{N}} \int_{[-\pi, \pi]^{N}} \frac{e^{-i j \cdot \xi}}{\tilde{\phi}(\xi)} d \xi .
$$

Now let $\phi_{j}:=\phi(j)$ satisfy the above with $\phi$ a piecewise polynomial function of compact support. With $\lambda \in l_{1}\left(Z^{N}\right)$ as defined above, set

$$
L(x)=\sum_{j} \lambda_{j} \phi(x-j) .
$$

We call $L$ the fundamental function associated with $\phi$ since

$$
L(j)=\delta_{0, j}, \quad j \in Z^{N} .
$$

The following proposition was observed in [3].

Proposition 2.1. L decays exponentially. More precisely, there exists a constant $A>0$, depending on $\phi$, so that

$$
|L(x)| \leqslant A \exp (-|x| / A), \quad x \in R^{N} .
$$

Using Lemma 1.1 and the above proposition, the following multivariate generalization of a well-known univariate result (cf. [12], [13]) can be easily established.

Proposition 2.2. Let $d \in l_{p}\left(Z^{N}\right), 1 \leqslant p \leqslant \infty$, and

$$
S(x)=\sum_{j \in Z^{N}} c_{j} \phi(x-j) \quad \text { with } c \in l_{p}\left(Z^{N}\right)
$$

be the function interpolating the data $d$; that is,

$$
S(j)=d_{j}, \quad j \in Z^{N} .
$$


Then

$$
S(x)=\sum_{j \in Z^{N}} d_{j} L(x-j),
$$

where the series is uniformly convergent on compact subsets of $\mathbf{C}^{N}$.

3. The Commutator of $\phi$. Motivated by the work of Frederickson [9], we introduce in this section the notion of the "commutator" of an ls-spline with a polynomial. For univariate $B$-splines with equally-spaced knots, it is equivalent to Marsden's identity [14, p. 125]. Proposition 3.1 will show that the commutator is also related to the order of approximation, in view of results of Strang and Fix [15] or Dahmen and Micchelli [8].

Throughout this section, $\phi: R^{N} \rightarrow R$ will denote a piecewise polynomial function with compact support, or ls-spline, and $P_{n}$ the space of polynomials in $N$ variables with total degree $n$. In order to avoid irregular cases, we shall also assume from now on that $\sum_{k \in Z^{N}} \phi(\cdot-k)=1$, in which case we call $\phi$ normalized. The properties of the map $f \rightarrow L_{\phi}(f)$, i.e., the properties of translates of $\phi$, have been considered in a bulk of papers (cf. [15], as an early reference, or [7] and the references therein). Here, we go one step further and consider the commutator of $\phi$ and a continuous function $f$, which we define by

$$
\begin{aligned}
{[\phi \mid f](x) } & :=\left(L_{\phi}(f)-L_{f}(\phi)\right)(x) \\
& =\sum_{k \in Z^{N}} \phi(x-k) f(k)-\sum_{k \in Z^{N}} f(x-k) \phi(k),
\end{aligned}
$$

for $x \in R^{N}$. The notion of the commutator of $\phi$ appears to be new even in the case of univariate $B$-splines $\phi$. if

Definition 3.1. The commutator of $\phi$ is said to be of order $m \in \mathbf{N}$ or degree $m-1$

$$
[\phi \mid f]=0 \text { for all } f \in P_{m-1} .
$$

Note that if $[\phi \mid f]=0$ for some polynomial $f$, we have a polynomial expressed as a linear combination of ls-splines.

We first wish to characterize those ls-spline functions $\phi$ with commutator of order $m$ while maintaining the assumption that $\phi$ is normalized. In the following result, the equivalence of conditions (iii) and (iv) have been derived by Strang and Fix [15, Theorem I]; they have shown that, equivalently, the approximation order by quasi-interpolation using translates of $\phi$ is equal to $\mathrm{m}$. Condition (ii) is due to de Boor (private communication) and its equivalence with (i) is essentially proved in [4].

We first remark that via the Poisson summation formula it is clear that $\phi$ is normalized if and only if $\hat{\phi}(0)=1$ and $\hat{\phi}(j)=0, j \neq 0$.

Proposition 3.1. Let $\phi$ be a normalized piecewise polynomial function with compact support. Then the following statements are equivalent for any $m \in \mathbf{N}$.

(i) The commutator of $\phi$ has order $m$.

(ii) For all $f \in P_{m-1}, L_{\phi}(f)$ is a polynomial.

(iii) For any $\alpha \in Z_{+}^{N}$ with $|\alpha|<m$ and $f(x)=x^{\alpha}$ the function $f-L_{\phi}(f)$ is a polynomial of degree $|\alpha|-1$.

(iv) $D^{\alpha} \hat{\phi}(2 \pi k)=0$ for all $k$ where $0 \neq k \in Z^{N}$ and $|\alpha|<m$ and $\hat{\phi}(0) \neq 0$. 
Proof. (iii) $\Leftrightarrow$ (iv): As mentioned above, this equivalence was derived by Strang and Fix [15, Theorem I].

(i) $\Rightarrow$ (ii) is trivial, since if $f$ is in $P_{m-1}, L_{f}(\phi)$ is a polynomial in $P_{|\alpha|}$ with leading term $\left[\sum_{k \in Z^{N}} \phi(k)\right] x^{\alpha}=x^{\alpha}$.

(ii) $\Rightarrow$ (i): Since $[\phi \mid f](i)={ }^{\prime} 0$ for all $i \in Z^{N}$, it follows that $\left.\left(L_{\phi}(f)-L_{f}(\phi)\right)\right|_{Z^{N}}$ $=0$, where both $L_{\phi}(f)$ and $L_{f}(\phi)$ are polynomials since $f$ is a polynomial. Hence,

$$
L_{\phi}(f) \equiv L_{f}(\phi) \text { or }[\phi \mid f]=0 .
$$

(i) $\Rightarrow$ (iii): This implication is obvious since $L_{f}(\phi)$ is a polynomial in $P_{|\alpha|}$ with leading term $\left[\sum_{k \in Z^{N}} \phi(k)\right] x^{\alpha}=x^{\alpha}$.

(iv) $\Rightarrow$ (i): If $\psi(x)=x^{\alpha} \phi(t-x)$, then, since

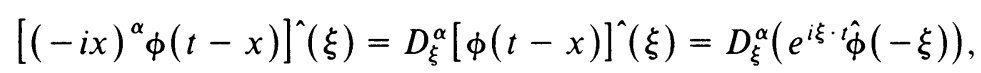

we obtain

$$
\begin{aligned}
\sum_{k} k^{\alpha} \phi(x-k) & =\left.\sum_{k} i^{|\alpha|} D_{\xi}^{\alpha}\left(e^{-i \xi \cdot \hat{\phi}}(-\xi)\right)\right|_{\xi=2 \pi k} \\
& =\sum_{\beta \leqslant \alpha}(-i)^{|\alpha|-|\beta|}\left(\begin{array}{l}
\alpha \\
\beta
\end{array}\right) x^{\beta} \sum_{k} e^{i 2 \pi k \cdot x} D^{\alpha-\beta} \hat{\phi}(-2 \pi k) .
\end{aligned}
$$

So if (iv) holds, then (3.3) implies that

$$
\sum_{k} k^{\alpha} \phi(x-k)=\sum_{\beta \leqslant \alpha}(-i)^{|\alpha|-|\beta|}\left(\begin{array}{l}
\alpha \\
\beta
\end{array}\right) D^{\alpha-\beta} \hat{\phi}(0) x^{\beta}
$$

for $|\alpha|<m$. On the other hand,

$$
\sum_{k} \phi(k)(x-k)^{\alpha}=\sum_{\beta \leqslant \alpha}(-1)^{|\alpha|-|\beta|}\left(\begin{array}{l}
\alpha \\
\beta
\end{array}\right) x^{\beta} \sum_{k} \phi(k) k^{\alpha-\beta},
$$

and Poisson's formula for $\Psi(x)=\phi(x) x^{\alpha-\beta}$ yields

$$
\begin{aligned}
\sum_{k} \phi(k) k^{\alpha-\beta} & =\sum_{k}\left(x^{\alpha-\beta} \phi(x)\right)^{\hat{(}}(2 \pi k) \\
& =i^{|\alpha|-|\beta|} \sum_{k} D^{\alpha-\beta} \hat{\phi}(2 \pi k)=i^{|\alpha|-|\beta|} D^{\alpha-\beta} \hat{\phi}(0)
\end{aligned}
$$

for $|\alpha-\beta|<m$. Hence,

$$
\sum_{k} k^{\alpha} \phi(x-k)=\sum_{k} \phi(k)(x-k)^{\alpha} \quad \text { for }|\alpha|<m .
$$

This completes the argument.

Remark. The same proof applies for the coordinate order $m \in Z^{N}$ of $\phi$, which is defined by $[\phi \mid f]=0$ for all $f(x)=x^{\alpha}$ with $\alpha_{i}<m_{i}, i=1, \ldots, N$. Here, statements (ii) and (iii) hold for $\alpha \in Z_{+}^{N}$ with $\alpha_{i}<m_{i}, i=1, \ldots, N$.

Since $(\phi * \Psi)=\hat{\phi} \hat{\Psi}$ where $f * g$ denotes the convolution of $f$ and $g$, the following is obtained as a corollary of Proposition 3.1 which, in case of Frederickson's triangular splines [9], is an equivalent formulation of his Lemma 6.1.

COROLlaRY 3.1. If $\phi$ and $\Psi$ are normalized piecewise polynomials with compact support and with commutator of order $m$ and $n$ respectively, then $\phi * \Psi$ is a normalized piecewise polynomial with compact support and with commutator of order $m+n$. 
Proof. Indeed,

$$
\left.D^{\alpha}(\hat{\phi}(\xi) \hat{\Psi}(\xi))\right|_{\xi=2 \pi k}=\sum_{\beta \leqslant \alpha}\left(\begin{array}{l}
\alpha \\
\beta
\end{array}\right) D^{\beta} \hat{\phi}(2 \pi k) D^{\alpha-\beta} \hat{\Psi}(2 \pi k)=0
$$

for $k \neq 0$ and $|\alpha|<m+n$, since $|\alpha|<m+n$ and $|\beta| \geqslant m$ implies $|\alpha-\beta|=|\alpha|-$ $|\beta|<n$; also, $\phi * \Psi$ is normalized since

$$
\sum_{k}(\phi * \Psi)(k)=\sum_{k} \hat{\phi}(2 \pi k) \hat{\Psi}(2 \pi k)=\hat{\phi}(0) \hat{\Psi}(0)=1 .
$$

Example 3.1. Let $I=\left[-\frac{1}{2}, \frac{1}{2}\right]$ and $\chi_{A}$ denotes, as usual, the characteristic function of $A$. With $M_{0}:=\chi_{I^{N}}, M_{m}:=M_{m-1} * \chi_{I^{N}}, m \geqslant 1$ we find that $M_{m}$ has a commutator of order $m+1$. For $N=1$ the recurrence relation above defines the symmetric cardinal $B$-splines; in case $N>1$ we have the corresponding tensor product splines. Note that

$$
\hat{M}_{m}\left(\zeta_{1}, \ldots, \zeta_{N}\right)=\prod_{i=1}^{N}\left(\sin \frac{\zeta_{i}}{2} / \frac{\zeta_{i}}{2}\right)^{m+1} .
$$

Example 3.2. The (symmetric) box splines may be introduced by their Fourier transform [2]. Given the fixed nonzero vectors $\xi_{1}, \ldots, \xi_{n} \in R^{N}$, then $\phi=M_{\xi_{1}, \ldots, \xi_{n}}$ is the inverse Fourier transform of the product function

$$
\hat{\phi}(\xi)=\prod_{i=1}^{n}\left[\sin \frac{\xi_{i} \cdot \xi}{2} / \frac{\xi_{i} \cdot \xi}{2}\right] \text {. }
$$

In case

$$
\xi_{1}, \ldots, \xi_{n}=\underbrace{e_{1}, \ldots, e_{1}}_{m+1}, \underbrace{e_{2}, \ldots, e_{2}}_{m+1}, \ldots, \underbrace{e_{N}, \ldots, e_{N}}_{m+1}, \quad n=N(m+1),
$$

we evidently get $\phi=M_{m}$ of the previous example; and according to Proposition 3.1, we have that the box spline $\phi=M_{\xi_{1}, \ldots, \xi_{n}}$ yields a commutator of order at least $m$ if $\hat{M}_{m-1}$ is a divisor of $\hat{\phi}$.

Example 3.3. An important special case of the previous example is the bivariate box spline (the Courant hat function) $\phi=B_{1,1,1}$, where

$$
\hat{B}_{1,1,1}(x, y)=\frac{\sin x / 2}{x / 2} \cdot \frac{\sin y / 2}{y / 2} \cdot \frac{\sin (x+y) / 2}{(x+y) / 2} .
$$

The commutator of $B_{1,1,1}$ clearly has order 2 . Thus Frederickson's bivariate box splines [9]

$$
B_{n, n, n-1}=M_{0} * B_{n-1, n-1, n-1}, \quad B_{n, n, n}=B_{1,1,1} * B_{n-1, n-1, n-1}, \quad n>1,
$$

yield a commutator of order $2 n-1$ and $2 n$, respectively.

We next observe how Marsden's identity for the univariate cardinal $B$-splines can be derived using the order of the commutator of the symmetric cardinal $B$-spline

$$
\phi(x)=M_{m-1}(x)=N_{0, m}(x+m / 2)
$$

with

$$
N_{0, m}(x)=m[0, \ldots, m](\cdot-x)_{+}^{m-1},
$$

where $\left[x_{1}, \ldots, x_{k}\right] f$ denotes, as usual, the divided difference of $f$ with respect to the knots $x_{1}, \ldots, x_{k}$. This motivates a Marsden's identity for multivariate splines. Recall from Example 3.1 above that $M_{m-1}$ has a commutator of order $m$. Now,

$$
x^{m-1}=\sum_{k \in Z} M_{m-1}\left(x+\frac{m}{2}-k\right) \prod_{r=1}^{m-1}(k-r)=: p_{m-1}(x),
$$


since $p_{0}(x)=1, p_{m-1}^{\prime}(x)=(m-1) p_{m-2}(x)$, and $p_{m-1}(0)=0$ for $m \geqslant 3$. Setting $f(x)=\prod_{r=1}^{m-1}(x-r)$ we obtain

$$
\begin{aligned}
(x-m / 2)^{m-1} & =L_{M_{m-1}}(f)(x)=L_{f}\left(M_{m-1}\right)(x) \\
& =\sum_{k} M_{m-1}(k) \prod_{r=1}^{m-1}(x+k-r),
\end{aligned}
$$

since $M_{m-1}$ is symmetric. With $x$ replaced by $y-x$ and $f_{y}(x):=\prod_{r=1}^{m-1}(y-x-r)$, we arrive at

$$
\begin{aligned}
(y-x)^{m-1} & =L_{f_{y}}\left(M_{m-1}\right)(x-m / 2)=L_{M_{m-1}}\left(f_{y}\right)(x-m / 2) \\
& =\sum_{k} N_{0, m}(x-k) \prod_{r=1}^{m-1}(y-k-r),
\end{aligned}
$$

which is Marsden's identity.

In extending this idea to the multivariate setting, we associate with $\phi$ the recursively defined polynomials: $g_{0}(x)=1$, and for $|\alpha|>0$,

$$
g_{\alpha}(x)=x^{\alpha}-\sum_{k \in Z^{N}} \phi(k) \sum_{\substack{\beta \leqslant \alpha \\
\beta \neq \alpha}}\left(\begin{array}{l}
\alpha \\
\beta
\end{array}\right)(-k)^{\alpha-\beta} g_{\beta}(x),
$$

and obtain the following result.

THEOREM 3.1. For a normalized piecewise polynomial function $\phi$ with compact support and $m \in \mathbf{N}$ the following statements are equivalent:

(i) The commutator of $\phi$ is of order $m$.

(ii) The polynomial reproducing formula

$$
x^{\alpha}=\sum_{j \in Z^{N}} g_{\alpha}(j) \phi(x-j) \quad \text { holds for }|\alpha|<m .
$$

Remark. In the univariate case, where $\phi(x)=M_{m-1}(x+m / 2)$ and $\alpha=m-1$, it follows that

$$
g_{m-1}(x)=\prod_{r=1}^{m-1}(x-r),
$$

since the representation of $x^{m-1}$ through translates of $\phi$ is unique in this case.

Proof of Theorem 3.1. There is nothing to prove in case $m=1$, since we assumed $\phi$ to be normalized. Now assume that the proposition has been proved with $m$ replaced by $m-1$. Since, for all $\alpha \in Z_{+}^{N}$,

$$
\begin{aligned}
\sum_{k \in Z^{N}} \phi(k)(x-k)^{\alpha} & =\sum_{k \in Z^{N}} \phi(k) \sum_{\beta \leqslant \alpha}\left(\begin{array}{l}
\alpha \\
\beta
\end{array}\right)(-k)^{\alpha-\beta} x^{\beta} \\
& =x^{\alpha}+\sum_{k \in Z^{N}} \phi(k) \sum_{\substack{\beta \leqslant \alpha \\
\beta \neq \alpha}}\left(\begin{array}{l}
\alpha \\
\beta
\end{array}\right)(-k)^{\alpha-\beta} x^{\beta},
\end{aligned}
$$


we may proceed in the induction step as follows. Suppose (i) holds. Then for $|\alpha|=m-1$, it follows that

$$
\begin{aligned}
\sum_{j \in Z^{N}} j^{\alpha} \phi(x-j) & =\sum_{k \in Z^{N}} \phi(k)(x-k)^{\alpha} \\
& =x^{\alpha}+\sum_{k \in Z^{N}} \phi(k) \sum_{\substack{\beta \leqslant \alpha \\
\beta \neq \alpha}}\left(\begin{array}{l}
\alpha \\
\beta
\end{array}\right)(-k)^{\alpha-\beta} \sum_{j \in Z^{N}} g_{\beta}(j) \phi(x-j)
\end{aligned}
$$

and

$$
\begin{aligned}
x^{\alpha} & =\sum_{j \in Z^{N}}\left\{j^{\alpha}-\sum_{k \in Z^{N}} \phi(k) \sum_{\substack{\beta \leqslant \alpha \\
\beta \neq \alpha}}\left(\begin{array}{l}
\alpha \\
\beta
\end{array}\right)(-k)^{\alpha-\beta} g_{\beta}(j)\right\} \phi(x-j) \\
& =\sum_{j \in Z^{N}} g_{\alpha}(j) \phi(x-j),
\end{aligned}
$$

so that (ii) also holds. On the other hand, in order to show (ii) $\Rightarrow$ (i), observe that

$$
\begin{aligned}
& \sum_{k \in Z^{N}} \phi(k)(x-k)^{\alpha} \\
& \quad=\sum_{j \in Z^{N}} g_{\alpha}(j) \phi(x-j)+\sum_{\substack{k \in Z^{N} \\
\phi(k)}} \sum_{\substack{\beta \leqslant \alpha \\
\beta \neq \alpha}}\left(\begin{array}{l}
\alpha \\
\beta
\end{array}\right)(-k)^{\alpha-\beta} \sum_{j \in Z^{N}} g_{\beta}(j) \phi(x-j) \\
& \quad=\sum_{j \in Z^{N}}\left\{g_{\alpha}(j)+\sum_{k \in Z^{N}} \phi(k) \sum_{\substack{\beta \leqslant \alpha \\
\beta \neq \alpha}}\left(\begin{array}{l}
\alpha \\
\beta
\end{array}\right)(-k)^{\alpha-\beta} g_{\beta}(j)\right\} \phi(x-j) \\
& =\sum_{j \in Z^{N}} j^{\alpha} \phi(x-j) .
\end{aligned}
$$

This concludes the proof.

Remark. Theorem 3.1 again holds for the coordinate order, in which case (ii) holds for $\alpha_{i}<m_{i}, i=1, \ldots, N$.

For the nontensor multidimensional box splines there are interesting examples of this recurrence relation. For instance, for a $C^{1}$ quadratic box spline $M$ on the four-direction mesh with directions $\left\{e_{1}, e_{2}, e_{1}+e_{2}, e_{1}-e_{2}\right\}$, where $e_{1}=(1,0)$ and $e_{2}=(0,1)$, we get for $k:=\left(k_{1}, k_{2}\right), x:=\left(x_{1}, x_{2}\right)$,

$$
\begin{aligned}
& \sum_{k} M(x-k)=1, \quad \sum_{k} k_{1} M(x-k)=x_{1}, \quad \sum_{k} k_{2} M(x-k)=x_{2}, \\
& \sum_{k}\left(k_{1}^{2}-1 / 4\right) M(x-k)=x_{1}^{2}, \quad \sum_{k}\left(k_{2}^{2}-1 / 4\right) M(x-k)=x_{2}^{2},
\end{aligned}
$$

and

$$
\sum_{k} k_{1} k_{2} M(x-k)=x_{1} x_{2} .
$$

More generally, if we have the directions

$$
\{\underbrace{e_{1}, \ldots, e_{1}}_{j}, \underbrace{e_{2}, \ldots, e_{2}}_{k}, \underbrace{e_{1}+e_{2}, \ldots, e_{1}+e_{2}}_{l}, \underbrace{e_{1}-e_{2}, \ldots, e_{1}-e_{2}}_{m}\},
$$


$n=j+k+l+m$, and $M$ is the corresponding box spline on the above four-directional mesh, we have, for $\alpha+\beta<r$,

$$
\begin{aligned}
x^{\alpha} y^{\beta}= & \sum_{\left(k_{1}, k_{2}\right) \in Z^{2}}\left(\sum_{w \leqslant(n+1) / 2}(-1)^{|w|} \sum_{\substack{\gamma+\theta=2 w \\
\gamma \leqslant \alpha ; \theta \leqslant \beta}} a_{\gamma, \theta} \frac{\alpha !}{(\alpha-\gamma) !} \frac{\beta !}{(\beta-\theta) !} k_{1}^{\alpha-\gamma} k_{2}^{\beta-\theta}\right) \\
& \cdot M\left(\left(x-k_{1}, y-k_{2}\right)\right),
\end{aligned}
$$

where

$$
a_{\gamma, 2 w-\gamma}=\sum_{t+2 p=\gamma} \sum_{u+v=w} \gamma_{2 p}^{j} \gamma_{2 v-2 p}^{k} \sum_{u+v=t}(-1)^{v} \sum_{v+s=u} \gamma_{2 v}^{l} \gamma_{2 s}^{r}\left(\begin{array}{c}
2 \gamma \\
u
\end{array}\right)\left(\begin{array}{c}
2 s \\
v
\end{array}\right)
$$

As a final connection along these lines we wish to relate the polynomials $g_{\alpha}$ derived in Theorem 3.1 and the quasi-interpolant operator used by Strang and Fix [15] and others, in order to derive their approximation orders.

If $\phi$ is normalized and has commutator of order at least one, then by Poisson's summation formula, $\hat{\phi}(0)=1$, so that $[\hat{\phi}(\xi)]^{-1}=\sum_{\beta \in Z_{+}^{N}} \alpha_{\beta} \xi^{\beta}$ is analytic at $\xi=0$. Define the quasi-interpolant for polynomials by

$$
(Q f)(x)=\sum_{k \in Z^{N}}\left(D_{\phi} f\right)(k) \phi(x-k)
$$

where

$$
\left(D_{\phi} f\right)=\sum_{\beta \in Z_{+}^{N}} \alpha_{\beta}\left(\frac{1}{i} D\right)^{\beta} f
$$

with $D=\left(\partial / \partial x_{1}, \ldots, \partial / \partial x_{N}\right)($ cf. [6]).

THEOREM 3.2. If the commutator of $\phi$ is of order $m$, then

$$
D_{\phi}\left(x^{\alpha}\right)=g_{\alpha}(x) \quad \text { for }|\alpha|<m \text {. }
$$

Proof. We show that both $g_{\alpha}$ and $D_{\phi}\left(x^{\alpha}\right)$ satisfy the same recurrence formulas for $|\alpha|<m$. For $\alpha=0$, it follows that

$$
D_{\phi}(1)=a_{0}=\frac{1}{\hat{\phi}(0)}=1=g_{0} \text {. }
$$

For $|\alpha|>0$ we have to show that for the case $|\alpha|<m$,

$$
x^{\alpha}=\sum_{k \in Z^{N}} \phi(k) \sum_{\beta \leqslant \alpha}\left(\begin{array}{l}
\alpha \\
\beta
\end{array}\right)(-k)^{\alpha-\beta} D_{\phi}\left(x^{\beta}\right) .
$$

The right-hand side is given by

$$
\begin{aligned}
\sum_{k \in Z^{N}} \phi(k) D_{\phi}\left(\sum_{\beta \leqslant \alpha}\left(\begin{array}{l}
\alpha \\
\beta
\end{array}\right)(-k)^{\alpha-\beta} x^{\beta}\right) & =D_{\phi}\left(\sum_{k \in Z^{N}} \phi(k)(x-k)^{\alpha}\right) \\
& =D_{\phi}\left(\sum_{k \in Z^{N}} k^{\alpha} \phi(x-k)\right),
\end{aligned}
$$

where the last equality holds using the order of the commutator. Now by Poisson's summation formula and Proposition 3.1,

$$
\sum_{k \in Z^{N}} k^{\alpha} \phi(x-k)=\sum_{\beta \leqslant \alpha}(-i)^{\alpha-\beta}\left(\begin{array}{l}
\alpha \\
\beta
\end{array}\right) D^{\alpha-\beta} \hat{\phi}(0) x^{\beta}
$$


since $D^{\alpha} \hat{\phi}(2 \pi k)=0$ for $|\alpha|<m$ and $0 \neq k \in Z^{N}$. Using the expansion $\hat{\phi}(\xi)=$ $\sum_{\beta \in Z_{+}^{N}} b_{\beta} \xi^{\beta}$, we get

$$
\begin{gathered}
D_{\phi}\left(\sum_{k \in Z^{N}} k^{\alpha} \phi(x-k)\right)=\sum_{\beta \leqslant \alpha}(-i)^{\alpha-\beta}\left(\begin{array}{l}
\alpha \\
\beta
\end{array}\right) D^{\alpha-\beta} \hat{\phi}(0) D_{\phi}\left(x^{\beta}\right) \\
=\sum_{\beta \leqslant \alpha}(-i)^{\alpha-\beta} \frac{\alpha !}{\beta !} b_{\alpha-\beta} \sum_{\gamma \leqslant \beta} a_{\gamma}(-i)^{\gamma} \frac{\beta !}{(\beta-\gamma) !} x^{\beta-\gamma} \\
=\sum_{\beta \leqslant \alpha}(-i)^{\alpha-\beta} \frac{\alpha !}{\beta !} b_{\alpha-\beta} \sum_{\gamma \leqslant \beta} a_{\beta-\gamma}(-i)^{\beta-\gamma} \frac{\beta !}{\gamma !} x^{\gamma} \\
=\sum_{\gamma \leqslant \alpha}(-i)^{\alpha-\gamma} \frac{\alpha !}{\gamma !} x^{\gamma} \sum_{\substack{\beta \\
\gamma \leqslant \beta \leqslant \alpha}} b_{\alpha-\beta} a_{\beta-\gamma}=x^{\alpha},
\end{gathered}
$$

since

$$
\sum_{\gamma \leqslant \beta \leqslant \alpha} b_{\alpha-\beta} a_{\beta-\gamma}=\sum_{\beta \leqslant \alpha-\gamma} b_{\alpha-\gamma-\beta} a_{\beta}= \begin{cases}1 & \text { if } \alpha-\gamma=0, \\ 0 & \text { otherwise, }\end{cases}
$$

which can be verified by equating coefficients in

$$
1=\hat{\phi}(\xi)[\hat{\phi}(\xi)]^{-1}=\sum_{\alpha} b_{\alpha} \xi^{\alpha} \sum_{\beta} a_{\beta} \xi^{\beta}=\sum_{j}\left(\sum_{\alpha+\beta=\gamma} b_{\alpha} a_{\beta}\right) \xi^{\gamma}
$$

The proof is complete.

This theorem yields an interesting result related to the work in [6]. In our case, the quasi-interpolator is given explicitly.

Corollary 3.1. Under the same assumptions as above, $Q f=f$ for all $f \in P_{m-1}$.

Proof. For $f(x)=x^{\alpha},|\alpha|<m$, it follows that

$$
Q f=\sum_{k}\left(D_{\phi} f\right)(k) \phi(\cdot-k)=\sum_{k} g_{\alpha}(k) \phi(\cdot-k)=x^{\alpha}
$$

according to the polynomial reproducing algorithm.

4. Approximation Orders Using Scaled Cardinal Interpolation. Various authors have dealt with approximation orders from translates of functions $\phi$ ([2], [4], [8], [9], [15]) using various quasi-interpolation methods. In view of the results in [4] and [8], this approximation order is the same for all $L_{p}$-norms, $1 \leqslant p \leqslant \infty$. It is therefore interesting to see that these orders can be obtained from the constructive method of scaled cardinal interpolation. This has already been pointed out by Bramble and Hilbert [5] in the tensor product case. We extend their results to the general case, and it again turns out that the notion of the commutator is central to the arguments.

For the rest of this section, the following assumptions based on Proposition 1.1 will be needed: $\phi: R^{N} \rightarrow R$ is a piecewise polynomial function with compact support which is

(i) nonnegative $(\phi(x) \geqslant 0$ for every $x)$,

(ii) normalized $\left(\sum_{k \in Z^{N}} \phi(\cdot-k)=1\right)$,

(iii) $\left|\sigma_{\phi}(z)\right| \geqslant C_{\phi}>0, z \in T^{N}$.

These assumptions will hereafter be referred to as Conditions A. 
Scaled cardinal interpolation refers to interpolation relative to the scaled multiintegers $Z_{h}^{N}:=h Z^{N}$, and the scaled function $\phi_{h}$ is given by $\phi_{h}(x):=\phi(x / h), h>0$. By analogy with the unscaled case, we say that the problem of scaled cardinal interpolation is $l_{p}$-solvable, if for any $v \in l_{p}\left(Z_{h}^{N}\right)$ there is a unique $u \in l_{p}\left(Z_{h}^{N}\right)$ satisfying

$$
\left.L_{\phi_{h}}(u)\right|_{Z_{h}^{N}}=v .
$$

The scaled discrete Fourier transformation is given by

$$
\tilde{u}(\xi)=h^{N} \sum_{j \in Z_{h}^{N}} u(j) e^{i j \cdot \xi}, \quad \xi \in R^{N},
$$

and hence the scaled symbol of $\phi$ satisfies

$$
\tilde{\phi}_{h}(\xi)=h^{N} \sum_{j \in Z_{h}^{N}} \phi_{h}(j) e^{i j \cdot \xi}=h^{N} \sum_{k \in Z^{N}} \phi(k) e^{i h k \cdot \xi}=h^{N} \tilde{\phi}(h \xi),
$$

where $\tilde{\phi}$ is the discrete Fourier transform of $\phi$. Thus, Proposition 1.1 can be applied and shows that, given

$$
v=\left.f\right|_{Z_{h}^{N}} \in l_{p}\left(Z_{h}^{N}\right),
$$

there is a unique $u \in l_{p}\left(Z_{h}^{N}\right)$ satisfying (4.1) and, correspondingly,

$$
S_{h}: f \rightarrow L_{\phi_{h}}(f)
$$

gives rise to an operator of scaled cardinal interpolation. Moreover, if $f \in C_{0}^{\infty}\left(\mathbf{R}^{N}\right)$, the subspace of $C^{\infty}\left(\mathbf{R}^{N}\right)$ of functions with compact support, then

(a) $S_{h}(f)^{\wedge}=\frac{\Phi_{\hat{h}}}{\phi_{\hat{h}}} \tilde{f}$ and

(b) $\left(S_{h}(f)-f\right)^{\wedge}=\frac{1}{\phi_{\hat{h}}}\left[\phi_{h} \mid f\right]^{\wedge}$,

where

$$
\begin{aligned}
{\left[\phi_{h} \mid f\right] } & =L_{\phi_{h}}(f)-L_{f}\left(\phi_{h}\right) \\
& =h^{N}\left\{\sum_{k \in Z_{h}^{N}} \phi_{h}(x-k) f(k)-\sum_{k \in Z_{h}^{N}} f(x-k) \phi_{h}(k)\right\} .
\end{aligned}
$$

We see that (a) above follows directly from [5, Theorem 8]. To see (b), note that by (a)

$$
\left(S_{h}(f)-f\right)^{\wedge}=\frac{\phi_{\hat{h}}}{\phi_{\hat{h}}} \tilde{f}-\hat{f}=\frac{1}{\phi_{\tilde{h}}}\left[\phi_{\hat{h}} \tilde{f}-\hat{f} \phi_{\tilde{h}}\right],
$$

while the equalities $\widehat{L_{f}(\phi)}=\hat{f} \tilde{\phi}$ and $\overline{L_{\phi}(f)}=\hat{\phi} \tilde{f}$ again follow from [5, p. 122]. Finally note that since

$$
\left[\phi_{h} \mid f\right](h x)=h^{N}[\phi \mid f(h \cdot)](x),
$$

the order of the commutator is invariant under scaling.

The following $L_{2}$-estimate (Theorem 4.1) for order of approximation by scaled interpolation is proved using ideas of [5], along with arguments that rely on the commutator. First recall the following fundamental theorem in [5, Theorem 2] where, for $1 \leqslant p<\infty, R$ is a bounded domain in $R^{N}$ satisfying the strong cone property, and $\rho$ is the diameter of $R$. The seminorms are defined by

$$
\|U\|_{p, R}^{p}=\frac{1}{\rho^{N}} \int_{R}|U(x)|^{p} d x, \quad|U|_{k, p, R}=\sum_{|\alpha|=k}\left\|D^{\alpha} U\right\|_{p, R},
$$


and

$$
\|U\|_{k, p, R}^{p}=\sum_{j=0}^{k} \rho^{j p}|U|_{j, p, R}^{p}
$$

THEOREM BH. Let $F$ be a linear functional on $H_{p}^{k}(R)$ which satisfies

(i) $|F(u)| \leqslant C\|u\|_{k, p, R}$ for all $u \in H_{p}^{k}(R)$ with $C$ independent of $\rho$ and $u$, and

(ii) $F(p)=0$ for all $p \in P_{k-1}$.

Then $|F(u)| \leqslant C_{1} \rho^{k}|u|_{k, p, R}$ for any $u \in H_{p}^{k}(R)$ with $C_{1}$ independent of $\rho$ and $u$.

We are now ready for the main result of this section. It generalizes [5, Theorem 9] which proved the result for tensor product splines.

THEOREM 4.1. Let $\phi$ satisfy Conditions (A). If $\phi$ has a commutator of order $m>N / 2$, then there exists a constant $\bar{K}>0$ so that

$$
\left\|S_{h}(f)-f\right\|_{2} \leqslant \bar{K} h^{m}|f|_{m, 2}, \quad f \in C_{0}^{\infty}\left(R^{N}\right),
$$

with

$$
|f|_{m, 2}^{2}=\sum_{|\alpha|=m}\left\|D^{\alpha} f\right\|_{2}^{2}=\sum_{|\alpha|=m} \int_{R^{N}}\left|D^{\alpha} f(x)\right|^{2} d x
$$

being the usual Sobolev seminorm of order $m$.

Proof. Using (4.2) and (4.3) together with Parseval's identity yields

$$
\left\|S_{h}(f)-f\right\|_{2}^{2}=\frac{1}{(2 \pi)^{N}}\left\|\left(S_{h}(f)-f\right)^{\wedge}\right\|_{2}^{2} \leqslant C_{\phi}^{-2} h^{-2 N}\left\|\left[\phi_{h} \mid f\right]\right\|_{2}^{2} .
$$

In order to estimate the right-hand side, we first consider

$$
F_{h}(f ; x):=h^{-N}\left[\phi_{h} \mid f\right](x)=\sum_{k \in Z_{h}^{N}}\left(\phi_{h}(x-k) f(k)-f(x-k) \phi_{h}(k)\right) .
$$

For fixed $x, F_{h}(f ; x)$ only depends on $\left.f\right|_{R_{x}}$ with

$$
R_{x}=x+h K\left[-\frac{1}{2}, \frac{1}{2}\right]^{N}
$$

where $K$ is chosen in such a way that

$$
\operatorname{supp}[\phi] \subseteq K\left[-\frac{1}{2}, \frac{1}{2}\right]^{N}
$$

We interpret $F_{h}(\cdot ; x)$ as a functional on the Sobolev space $H_{2}^{m}\left(R_{x}\right)$ and apply Theorem BH.

Clearly, $F_{h}(p ; x)=0$ for polynomials $p \in P_{m-1}$, because of the order of the commutator, and

$$
\left|F_{h}(f ; x)\right| \leqslant \sup _{t \in R_{x}}|f(y)| \sum_{k \in Z_{h}^{N}}\left(\left|\phi_{h}(x-k)\right|+\left|\phi_{h}(k)\right|\right)=2 \sup _{y \in R_{x}}|f(y)| .
$$

The last equality follows since $\phi$ is nonnegative and normalized, and the commutator of $\phi$ is of order at least one. Hence,

$$
\begin{aligned}
\sum_{k \in Z_{h}^{N}}\left|\phi_{h}(x-k)\right| & =\sum_{k \in Z_{h}^{N}} \phi_{h}(x-k)=\sum_{k \in Z_{h}^{N}} \phi\left(\frac{x-k}{h}\right) \\
& =\sum_{k \in Z^{N}} \phi\left(\frac{x}{h}-k\right)=\sum_{k \in Z^{N}} \phi(k)=1 .
\end{aligned}
$$

Now (4.3) together with Sobolev's lemma [1, p. 32] implies that

$$
\left|F_{h}(f ; x)\right| \leqslant C\|f\|_{m, 2, R_{x}},
$$


since $m>N / 2$, where the constant $C$ is independent of $x$ and $h$. Since the diameter $R$ is given by $\rho=h K \sqrt{N}$, Theorem BH yields the pointwise estimate

$$
\left|F_{h}(f ; x)\right| \leqslant C^{\prime} h^{m}|f|_{m, 2, R_{x}}
$$

with the constant $C^{\prime}$ independent of $x$ and $h$.

The proof is completed by writing $|f|_{m, 2, R_{x}}^{2}$ as a convolution, namely,

$$
\begin{aligned}
|f|_{m, 2, R_{x}}^{2} & =\sum_{|\alpha|=m} \frac{1}{(h K \sqrt{N})^{N}} \int_{R_{x}}\left|D^{\alpha} f(y)\right|^{2} d y \\
& =\sum_{|\alpha|=m} \frac{1}{(h K \sqrt{N})^{N}} \int_{R_{0}}\left|D^{\alpha} f(x-y)\right|^{2} d y \\
& =\frac{1}{(\sqrt{N})^{N}} \sum_{|\alpha|=m}\left(g_{R_{0}} *\left|D^{\alpha} f\right|^{2}\right)(x),
\end{aligned}
$$

with

$$
g_{R_{0}}(y)= \begin{cases}\frac{1}{(h K)^{N}} & \text { for } y \in h K\left[-\frac{1}{2}, \frac{1}{2}\right]^{N}=R_{0} \\ 0 & \text { otherwise. }\end{cases}
$$

Since $\int_{R^{N}}\left|g_{R_{0}}(y)\right| d y=1$, we finally obtain

$$
\begin{aligned}
h^{-2 N}\left\|\left[\phi_{h} \mid f\right]\right\|_{2}^{2} & =\int_{R^{N}}\left|F_{h}(f ; x)\right|^{2} d x \leqslant M h^{2 m} \int_{R^{N}}|f|_{m, 2, R_{x}}^{2} d x \\
& \leqslant \tilde{M} h^{2 m} \sum_{|\alpha|=m} \int_{R^{N}}\left(g_{R_{0}} *\left|D^{\alpha} f\right|^{2}\right)(x) d x \\
& \leqslant \tilde{M} h^{2 m} \sum_{|\alpha|=m}\left\|D^{\alpha} f\right\|_{2}^{2}=\tilde{M} h^{2 m}|f|_{m, 2}^{2},
\end{aligned}
$$

where $M$ and $\tilde{M}$ are constants. This completes the proof of the theorem.

Acknowledgment. We would like to thank Professor Carl de Boor and the referee for their helpful suggestions and comments that improved this version of the manuscript substantially. We would also like to thank Mr. M. J. Lai for helping us on several computations. In addition, the second author wishes to thank the mathematics department at Texas A \& M University and, in particular, the members of the Center for Approximation Theory, for making his stay at Texas A\& M possible.

Department of Mathematics Texas A \& M University College Station, Texas 77843

Department of Mathematics University of Duisburg

Duisburg, Federal Republic of Germany

Department of Mathematics Texas A \& M University College Station, Texas 77843 
1. S. AGmon, Lectures on elliptic boundary value problems, Van Nostrand, Princeton, N. J., 1965.

2. C. DE Boor \& K. Höllig, “B-splines from parallelepipeds," J. Analyse Math., v. 42, 1983, pp. 99-115.

3. C. DE Boor, K. Höllig \& S. Riemenschneider, "Bivariate cardinal interpolation by splines on a three direction mesh,” Illinois J. Math., v. 29, 1985, pp. 533-566.

4. C. DE BOOR \& R.-Q. JIA, "Controlled approximation and a characterization of the local approximation order," Proc. Amer. Math. Soc., v. 95, 1985, pp. 547-553.

5. J. H. Bramble \& S. R. Hilbert, "Estimation of linear functionals on Sobolev spaces with application to Fourier transforms and spline interpolation," SIAM J. Numer. Anal., v. 7, 1970, pp. 112-124.

6. W. Dahmen \& C. A. Micchelli, "Translates of multivariate splines," Linear Algebra Appl., v. $52 / 53,1983$, pp. 217-234.

7. W. Dahmen \& C. A. Micchel.li, "Recent progress in multivariate splines," in Approximation Theory IV (C. K. Chui, L. L. Schumaker \& J. D. Ward, eds.), Academic Press, New York, 1983, pp. 27-121.

8. W. Dahmen \& C. A. Micchelli, "On the approximation order from certain multivariate spline spaces,” J. A ustral. Math. Soc., v. 26, 1984, pp. 233-246.

9. P. O. Frederickson, Generalized Triangular Splines, Mathematics Report 7-71, Lakehead Univ., 1971.

10. W. Rudin, Functional Analysis, McGraw-Hill, New York, 1973.

11. I. J. Schoenberg, Cardinal Spline Interpolation, SIAM Publ., Philadelphia, Pa., 1973.

12. I. J. Schoenberg, “Cardinal interpolation and spline functions," J. Approx. Theory, v. 2, 1969, pp. $167-206$.

13. I. J. Schoenberg, "Cardinal interpolation and spline functions: II. Interpolation of data of power growth,” J. Approx. Theory, v. 6, 1972, pp. 404-420.

14. L. L. Schumaker, Spline Functions: Basic Theory, Wiley, New York, 1981.

15. G. Strang \& G. Fix, “A Fourier analysis of the finite element variational method,” C.I.M.E. II Cilo 1971, Constructive Aspects of Functional Analysis (G. Geymonat, ed.), 1973, pp. 793-840. 